\title{
Synthesis and Characterization of Luminescent Polyfluorenes Incorporating Side-Chain-Tethered Polyhedral Oligomeric Silsesquioxane Units
}

\author{
Chia-Hung Chou, So-Lin Hsu, K. Dinakaran, Mao-Yuan Chiu, and Kung-Hwa Wei* \\ Department of Materials Science and Engineering, National Chiao Tung University, \\ Hsinchu, Taiwan 30049 R.O.C. \\ Received October 4, 2004; Revised Manuscript Received November 8, 2004
}

\begin{abstract}
Using Suzuki polycondensation, we have synthesized polyhedral silsesquioxane-tethered polyfluorene copolymers, poly $\left(9,9^{\prime}\right.$-dioctylfluorene-co-9, $9^{\prime}$-bis [4-( $N, N$-dipolysilsesquioxane)aminophenyl]fluorene) (PFO-POSS), that have well-defined architectures. This particular PFO-POSS molecular architecture increases the quantum yield of polyfluorene significantly by reducing the degree of interchain aggregation; in addition, these copolymers exhibit a purer and stronger blue light by preventing the formation of keto defects.
\end{abstract}

\section{Introduction}

Semiconducting polymers have been studied extensively for their potential applications in electroluminescent displays, ${ }^{1}$ solar cells, ${ }^{2}$ and thin film organic transistors. ${ }^{3}$ One of the most promising conjugated polymers is polyfluorene, which has been applied widely for its characteristic of emitting blue light. ${ }^{4-7}$ Polyfluorenes may be functionalized readily by modifying the C-9 position of the fluorene monomer; such modification can, for instance, provide good solubility in common organic solvents that allows further processing. In the solid state, polyfluorene and its derivatives exhibit adequate photoluminescence (PL) and electroluminescence (EL) efficiencies. ${ }^{8-10}$ The application of polyfluorenes in light emitting diodes, however, has been hampered by the appearance of green electroluminescence (ca. $530 \mathrm{~nm}$ ) in addition to the desired peak at $425 \mathrm{~nm}$; this green electroluminescence has been attributed to either intermolecular interactions, which lead to the formation of aggregates, ${ }^{11-17}$ or to the presence of emissive keto defect sites that arise as a result of thermo- or electrooxidative degradation of the polyfluorene backbone. ${ }^{18 a, b}$ A detailed discussion of this phenomenon has been presented elsewhere. ${ }^{18 c}$ As a result, the expected blue emission from polyfluorene becomes an undesired bluegreen color in LED applications.

Several approaches have been adopted to reduce the formation of aggregation or keto defect in polyfluorenes, including the introduction of bulky side chains, using cross-linked structures, improving the oxidative stability of pendant groups or chain ends, ${ }^{15}$ and limiting chain mobility by blending it with a high- $T_{\mathrm{g}}$ polymer. ${ }^{17}$ One of the most recent approaches involves incorporation of polyhedral oligomeric silsesquioxane (POSS) into the conjugated polymer. The first such study was undertaken by attaching POSS covalently to the chain ends of poly(2-methoxy-5-[2-ethylhexyloxy]-1,4-phenylenevinylene) (MEHPPV) and poly (9,9'-dioctylfluorene) (PFO), which resulted in enhanced thermal stability of the devices prepared from these modified polymers. ${ }^{15}$ The density of the polymer chain ends, however, decreases

\footnotetext{
* Corresponding author. Telephone: 886-35-731871. Fax: 886-35-724727. E-mail: khwei@cc.nctu.edu.tw.
}

as the molecular weight of the polymer increases, which limits the amount of POSS that can be attached (ca. $1.2 \%)$. Another study involved a synthesis of a bridged polyfluorene copolymer by using fluorene tetrabromide monomers featuring a siloxane bridge. The thermal stability of a device made from this siloxane-bridged polyfluorene appears to be better than that prepared from pure PFO. ${ }^{18 d}$ A third related study involved attaching polyfluorene to the functionalized vertexes on the cubic polyhedral silsesquioxane core units to form starlike structures; these polymers possessed improved thermal and optoelectronic characteristics. The amount of POSS content in the polyfluorene was ca. $3.8 \% .{ }^{18 \mathrm{e}}$ The enhanced electroluminescence characteristics in these polyfluorenes have been attributed to POSS imparting a reduction in either the degree of aggregation and excimer formation or the number of keto defects. The molecular architecture that polyfluorene possessed in the latter two studies cannot be defined easily. Previously, we synthesized a polyimide-sidechain-tethered POSS as an approach to lowering its dielectric constant. ${ }^{19 a}$ In this present study, we took a copolymer approach by synthesizing polyfluorenetethered POSS in a well-defined architecture. To the best of our knowledge, the introduction of an inorganic side group, such as POSS, into the C-9 position of polyfluorene has not been explored previously. We believe that by developing a POSS/polyfluorene copolymer having well-defined architecture we will be able to tailor its luminescence properties more precisely by modifying the molecular structure. In this paper, we report the synthesis and characterization of fluorenebased random copolymers featuring tethered polyhedral oligomeric silsesquioxanes (POSS) units.

Scheme 1 displays the synthetic procedure we used to prepare the POSS-dibromide monomer (D-POSS-diAF), in which cyclopentyl-POSS was covalently bonded to the C-9 carbon atom of the fluorene unit through a 4-aminophenyl spacer. We used POSS-dibromide as a comonomer, which, together with 2,7-dibromo-9,9'-dioctylfluorenone (5), were reacted with 2,7-bis $(4,4,5,5$-tetramethyl-1,3,2-dioxaborolan-2yl)fluorene (6) through Suzuki coupling followed by end capping. Scheme 2 presents the complete synthetic 
Scheme 1. Synthesis of D-POSS-diAF (4) ${ }^{a}$
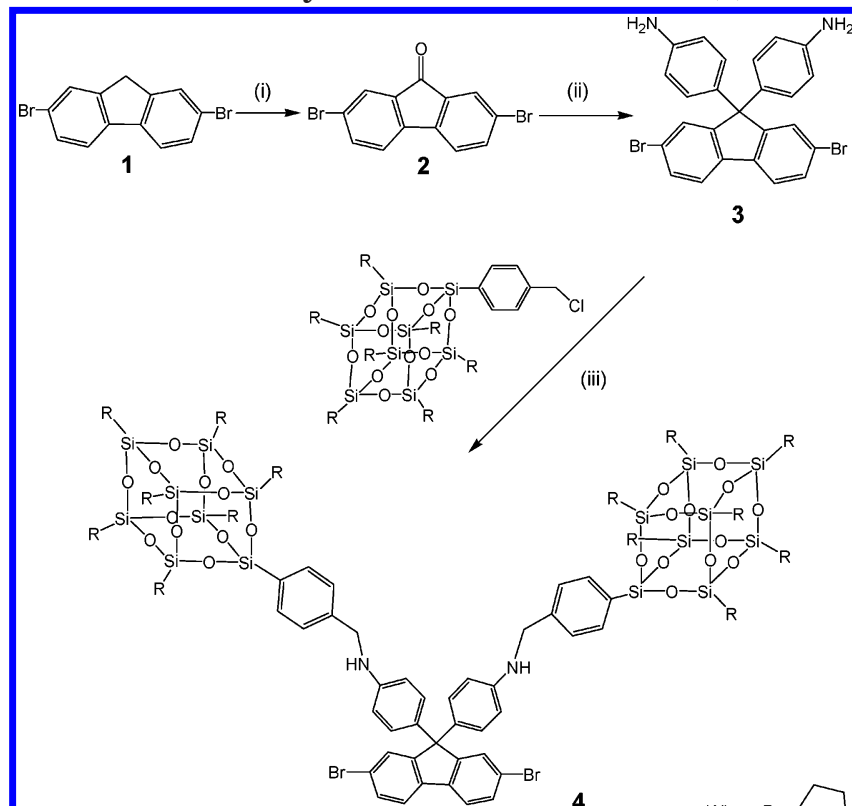

Where $\mathrm{R}=$

${ }^{a}$ (i) $\mathrm{CrO}_{3}$, acetic anhydride, $\mathrm{HCl}_{\mathrm{aq}}$. (ii) aniline, aniline hydrochloride. (iii) $\mathrm{K}_{2} \mathrm{CO}_{3}, \mathrm{KI}, \mathrm{DMF} / \mathrm{THF}(5: 4)$.

procedure for the preparation of POSS-tethered polyfluorene. The extended 9,9'-bis(4-aminophenyl)fluorenyl core, which is readily accessible, offers the following additional advantages: (1) Direct attachment of the benzyl groups of POSS to the C-9 carbon atom of the fluorene unit is avoided; such benzyl linkages are potentially susceptible to photooxidation, which may cause degradation and failure of the polymer LEDs. ${ }^{19 b, 20}$ (2) The introduction of the 4-aminophenyl spacer reduces the steric hindrance imposed by POSS. ${ }^{21}$ The insertion of a rigid phenylene spacer between the POSS side chain and the polymer backbone may lead to a shielding effect on the polyfluorene main chain, while leaving the reaction sites of the macromonomer accessible for the palladium-catalyzed polymerization reaction. (3) Through this copolymerization approach, the amount of POSS incorporated into the polyfluorene may be tuned by controlling the amount of POSSdibromide monomer used in the polymerization.

\section{Experimental Section}

Materials. 2,7-Dibromo-9,9'-dioctylfluorenone (5), ${ }^{21}$ 2,7-bis(4,4,5,5-tetramethyl-1,3,2-dioxaborolan-2-yl)fluorene $(\mathbf{6}){ }^{22}$ and the chlorobenzylcyclopentyl-POSS ${ }^{23}$ were synthesized according to literature procedures. THF was distilled under nitrogen from sodium benzophenone ketyl; other solvents were dried using standard procedures. All other reagents were used as received from commercial sources unless otherwise stated.

Chlorobenzylcyclopentyl-POSS. ${ }^{1} \mathrm{H}$ NMR $(300 \mathrm{MHz}$, $\left.\mathrm{CDCl}_{3}\right): \delta 7.64(\mathrm{~d}, J=8.1 \mathrm{~Hz}, 2 \mathrm{H}), 7.37(\mathrm{~d}, J=8.1 \mathrm{~Hz}, 2 \mathrm{H})$, $4.57(\mathrm{~s}, 2 \mathrm{H}), 2.26-1.21(\mathrm{~m}, 56 \mathrm{H}), 1.16-0.81(\mathrm{~m}, 7 \mathrm{H}) \mathrm{ppm} .{ }^{29} \mathrm{Si}$ NMR (600 MHz, THF): $\delta-67.8,-68.2,-79.6 \mathrm{ppm}$.

Synthesis of 9,9'-Bis(4-aminophenyl)-2,7-dibromofluorene (3). A mixture of 2,7-dibromo-9-fluorenone (2) ( $3.0 \mathrm{~g}, 8.88$ $\mathrm{mmol})$, aniline $(4.0 \mathrm{~g}, 4.30 \mathrm{mmol})$, and aniline hydrochloride (1.15 g, $8.88 \mathrm{mmol}$ ) was heated at $150{ }^{\circ} \mathrm{C}$ under nitrogen for 6 $\mathrm{h}$. The reaction mixture was then slowly added into water (150 $\mathrm{mL})$ and extracted with ethyl acetate $(3 \times 50 \mathrm{~mL})$. The combined extracts were dried $\left(\mathrm{MgSO}_{4}\right)$, the solvent was evaporated, and the residue was purified by column chromatography (hexane/ethyl acetate, $4: 1)$ to afford 3 (3.23 g, 72\%). ${ }^{1} \mathrm{H}$ NMR $\left(\mathrm{CDCl}_{3}\right): \delta 7.52(\mathrm{~d}, J=8.1 \mathrm{~Hz}, 2 \mathrm{H}), 7.44(4 \mathrm{H}), 6.90$ $(\mathrm{d}, J=8.1 \mathrm{~Hz}, 4 \mathrm{H}), 6.53(\mathrm{~d}, J=8.1 \mathrm{~Hz}, 4 \mathrm{H}), 3.58(\mathrm{~s}, 4 \mathrm{H}) \mathrm{ppm}$.
Anal. Calcd for $\mathrm{C}_{25} \mathrm{H}_{18} \mathrm{Br}_{2} \mathrm{~N}_{2}$ : C, 59.31; H, 3.58; N, 5.53. Found: C, 59.38; H, 3.62; N, 5.47.

Synthesis of 9,9'-Bis[4-( $N, \boldsymbol{N}$-dipolysilsesquioxane)aminophenyl]fluorene (D-POSS-diAF) (4). 9,9-Bis(4-aminophenyl)-2,7-dibromofluorene ( $200 \mathrm{mg}, 0.395 \mathrm{mmol}$ ) was stirred with $\mathrm{K}_{2} \mathrm{CO}_{3}(764 \mathrm{mg}, 5.53 \mathrm{mmol})$ and $\mathrm{KI}(262 \mathrm{mg}, 1.58 \mathrm{mmol})$ in DMF ( $5 \mathrm{~mL}$ ) and THF $(4 \mathrm{~mL})$ at room temperature for $1 \mathrm{~h}$. A small amount of Cl-POSS (888 mg, $0.832 \mathrm{mmol}$ ) was added and then the whole mixture was heated at $70{ }^{\circ} \mathrm{C}$ for $3 \mathrm{~h}$. The reaction mixture was then slowly poured into water $(150 \mathrm{~mL})$ and extracted with chloroform $(3 \times 30 \mathrm{~mL})$. The combined extracts were dried $\left(\mathrm{MgSO}_{4}\right)$, the solvents were evaporated, and the residue was purified by column chromatography (hexane/ chloroform, 1:10) to afford $4(0.69 \mathrm{~g}, 68 \%) .{ }^{1} \mathrm{H}$ NMR $\left(\mathrm{CDCl}_{3}\right): \delta 7.62-7.63(\mathrm{~m}, 14 \mathrm{H}), 7.01-6.84(\mathrm{~m}, 8 \mathrm{H}), 4.81(\mathrm{~s}$, $2 \mathrm{H}), 4.27(\mathrm{~s}, 4 \mathrm{H}), 2.06-1.18(\mathrm{~m}, 112 \mathrm{H}), 1.14-0.79(\mathrm{~m}, 14 \mathrm{H})$ ppm. Anal. Calcd for $\mathrm{C}_{109} \mathrm{H}_{154} \mathrm{Br}_{2} \mathrm{O}_{24} \mathrm{~N}_{2} \mathrm{Si}_{16}$ (\%): C, 52.67; H, 6.24; N, 1.13. Found: C, 52.11; H, 6.21; N, 1.07 .

General Procedure for the Synthesis of Alternating Copolymers PFO-POSS. Aqueous potassium carbonate (2 M) and aliquate 336 were added to a solution of the POSSappended fluorene dibromide monomer $\mathbf{4}$, dibromide $\mathbf{5}$, and diboronate $\mathbf{6}$ in toluene. The mixture was degassed and purged with nitrogen three times. The catalyst, tetrakis(triphenylphosphine)palladium (3.0 mol \%), was added in one portion under a nitrogen atmosphere. The solution was then heated at 90 ${ }^{\circ} \mathrm{C}$ and vigorously stirred under nitrogen for 5 days. End group capping was performed by heating the solution under reflux for $6 \mathrm{~h}$ sequentially with phenylboronic acid and bromobenzene. After cooling, the polymer was recovered by precipitating it into a mixture of methanol and acetone (4:1). The crude polymer was collected, purified twice by reprecipitation from THF into methanol, and subsequently dried under vacuum at $50{ }^{\circ} \mathrm{C}$ for $24 \mathrm{~h}$. The ${ }^{1} \mathrm{H}$ and ${ }^{13} \mathrm{C}$ NMR spectra of PFO and PFO-POSS appear to be identical because of the low content of POSS in the latter polymer.

Characterization. ${ }^{1} \mathrm{H},{ }^{13} \mathrm{C}$, and ${ }^{29} \mathrm{Si}$ nuclear magnetic resonance (NMR) spectra of the compounds were obtained using a Bruker DRX $300 \mathrm{MHz}$ spectrometer. Mass spectra of the samples were obtained on a JEOL JMS-SX 102A spectrometer. Fourier transform infrared (FTIR) spectra of the synthesized materials were acquired using a Nicolet 360 FT-IR spectrometer. Gel permeation chromatographic analyses were performed on a Waters 410 Differential refractometer and a Waters 600 controller (Waters Styragel column). All GPC analyses of polymers in THF solutions were performed at a flow rate of $1 \mathrm{~mL} / \mathrm{min}$ at $40{ }^{\circ} \mathrm{C}$; the samples were calibrated using polystyrene standards. Thermogravimetric analysis (TGA) and differential scanning calorimetry (DSC) measurements were performed under a nitrogen atmosphere at heating rates of 20 and $10{ }^{\circ} \mathrm{C} / \mathrm{min}$, respectively, using Du Pont TGA2950 and TA-2000 instruments, respectively. UV-vis absorption and photoluminescence (PL) spectra were recorded on a HP 8453 spectrophotometer and a Hitachi F-4500 luminescence spectrometer, respectively. Before investigating the thermal stability of the synthesized polymers, their polymer films were annealed in air at $200{ }^{\circ} \mathrm{C}$ for $2 \mathrm{~h}$.

Device Fabrication and Testing. The electroluminescent (EL) devices were fabricated on an ITO-coated glass substrate that was precleaned and then treated with oxygen plasma before use. A layer of poly(ethylene dioxythiophene):poly(styrenesulfonate) (PEDOT:PSS, Baytron P from Bayer Co.; ca. $40 \mathrm{~nm}$ thick) was formed by spin-coating from its aqueous solution $(1.3 \mathrm{wt} \%)$. The EL layer was spin-coated at $1500 \mathrm{rpm}$ from the corresponding toluene solution $\left(15 \mathrm{mg} \mathrm{mL}^{-1}\right)$ on top of the vacuum-dried PEDOT:PSS layer. The nominal thickness of the EL layer was $65 \mathrm{~nm}$. Using a base pressure below $1 \times$ $10^{-6}$ Torr, a layer of $\mathrm{Ca}(30 \mathrm{~nm})$ was vacuum deposited as the cathode and a thick layer of $\mathrm{Al}$ was deposited subsequently as the protecting layer. The current-voltage characteristics were measured using a Hewlett-Packard 4155B semiconductor parameter analyzer. The power of the EL emission was measured using a Newport 2835-C multifunction optical meter. The brightness was calculated using the forward output power 
Scheme 2. Synthesis of PFO-POSS Copolymers

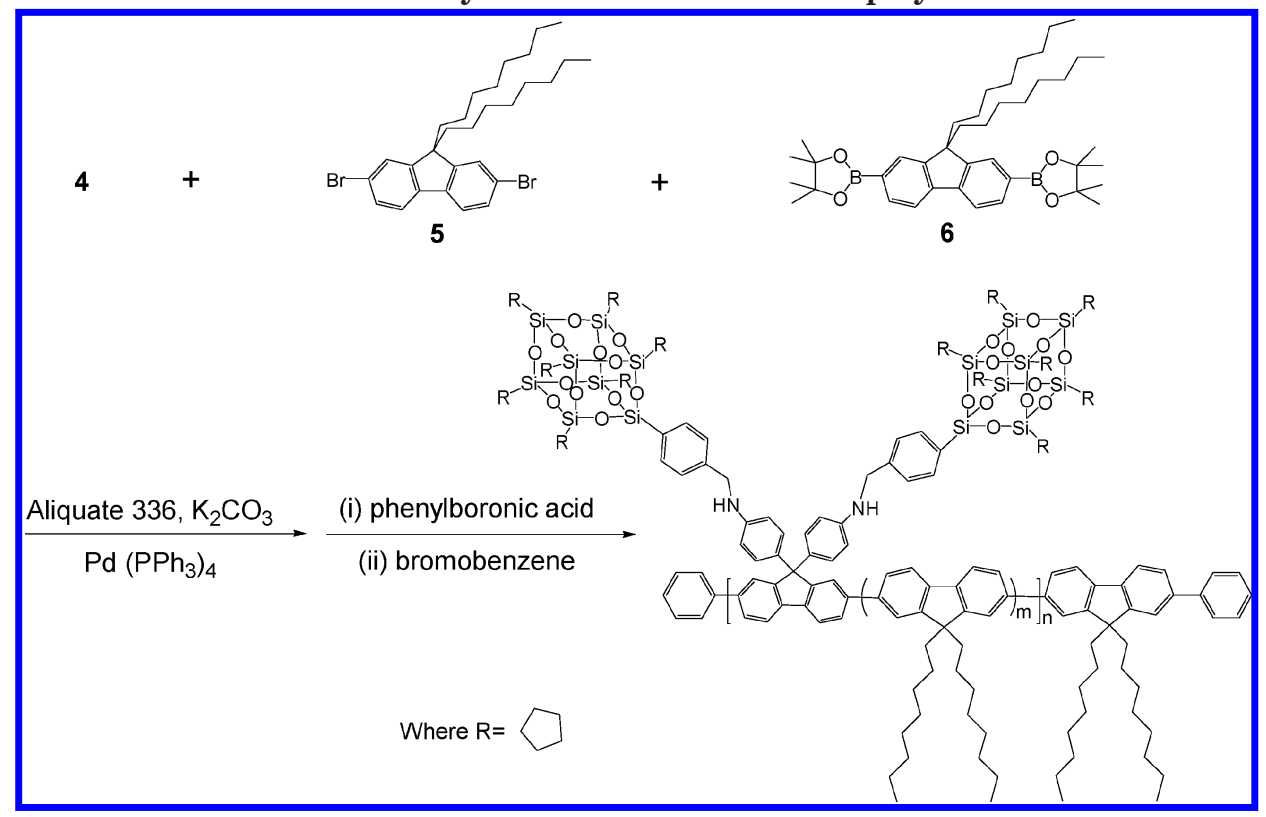

and the EL spectra of the devices; a Lambertian distribution of the EL emission was assumed.

\section{Results and Discussion}

Figure 1 displays the ${ }^{1} \mathrm{H}$ NMR spectra of 9,9'-bis(4aminophenyl)-2,7-dibromofluorene (3), Cl-POSS, and D-POSS-diAF (4). The peak for the NH protons shifted downfield from 3.59 ppm for 3 to 4.48 ppm for 4, while the $\mathrm{CH}_{2}$ peak of $\mathbf{C l}$-POSS shifted upfield from 4.47 to $4.21 \mathrm{ppm}$ in D-POSS-diAF. The ratio of the peak areas of the benzylic $\mathrm{CH}_{2}$ and $\mathrm{NH}$ protons is ca. $2: 1$. Taken together, all of these data suggest that Cl-POSS had reacted with 4-aminophenyl fluorene to form D-POSS-diAF. Table 1 lists the thermal properties and molecular weight distributions of the PFO-POSS copolymers. Both the thermal degradation and glass transition temperature increased as the amount of POSS in PFO increased, presumably because the tethered POSS enhanced the thermal stability and retarded the polymer chain mobility. The molecular weights of the PFO-POSS copolymers decreased upon increasing the POSS content; this phenomenon can be attributed to the steric hindrance caused by POSS during the polymerization process.

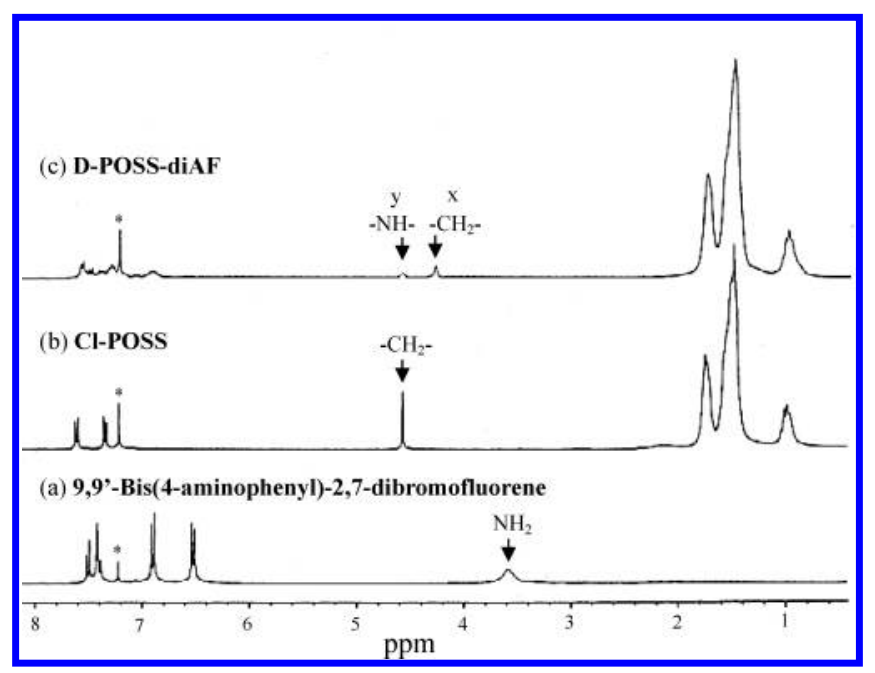

Figure 1. ${ }^{1} \mathrm{H}$ NMR spectra of (a) 3, (b) Cl-POSS, and (c) 4.
Table 1. Physical Properties of the PFO-POSS Copolymers

\begin{tabular}{lccccc}
\hline & $T_{\mathrm{d}}{ }^{a}\left({ }^{\circ} \mathrm{C}\right)$ & $M_{\mathrm{n}}$ & $M_{\mathrm{w}}$ & PDI & yield $(\%)$ \\
\hline PFO & 372 & 27000 & 51000 & 1.86 & 96 \\
PFO-POSS-1\% & 382 & 21000 & 42000 & 1.96 & 78 \\
PFO-POSS-3\% & 381 & 20000 & 37000 & 1.85 & 68 \\
PFO-POSS-5\% & 397 & 16000 & 31000 & 1.93 & 79 \\
PFO-POSS-10\% & 416 & 12000 & 24000 & 1.97 & 65
\end{tabular}

${ }^{a}$ Temperature at which $5 \%$ weight loss occurred, based on the initial weight.

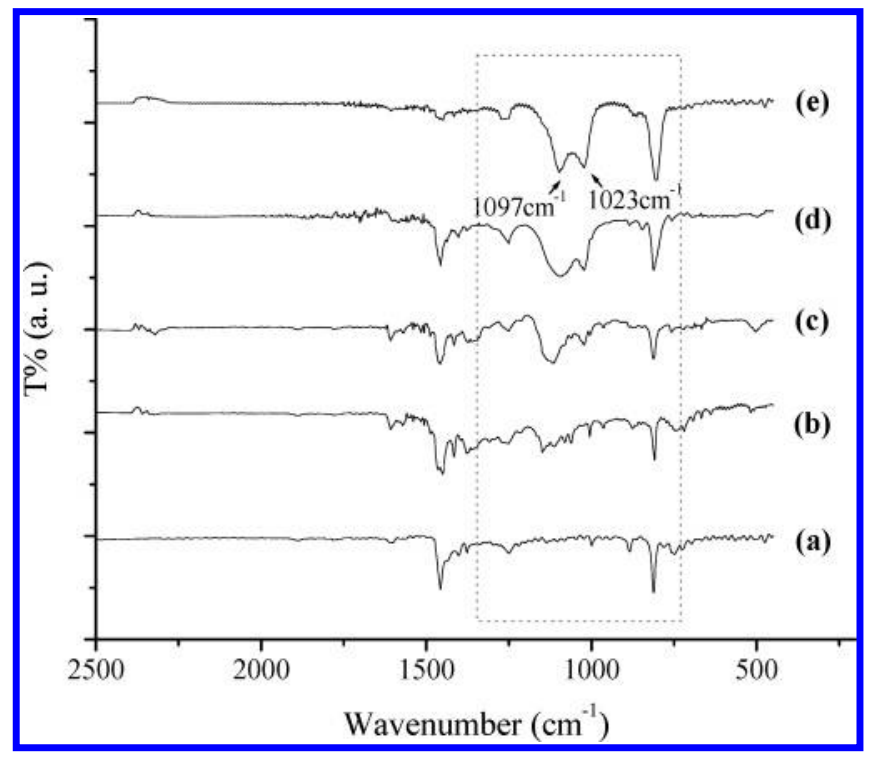

Figure 2. FTIR spectra of (a) PFO, (b) PFO-POSS-1\%, (c) PFO-POSS-3\%, (d) PFO-POSS-5\%, and (e) PFO-POSS$10 \%$.

Figure 2 displays FTIR spectra of PFO copolymers containing different amounts of POSS. The FTIR spectrum of POSS displays two major characteristic peaks in the range $1000-1180 \mathrm{~cm}^{-1}(\mathrm{Si}-\mathrm{O}-\mathrm{Si}$ stretching). The $\mathrm{Si}-\mathrm{C}$ band at $1074 \mathrm{~cm}^{-1}$, however, overlaps with the $\mathrm{Si}-\mathrm{O}-\mathrm{Si}$ band and, thus, could not be observed clearly.

Figure 3 displays the X-ray diffraction curves of Cl-POSS, PFO, and PFO-POSS. We observed no 


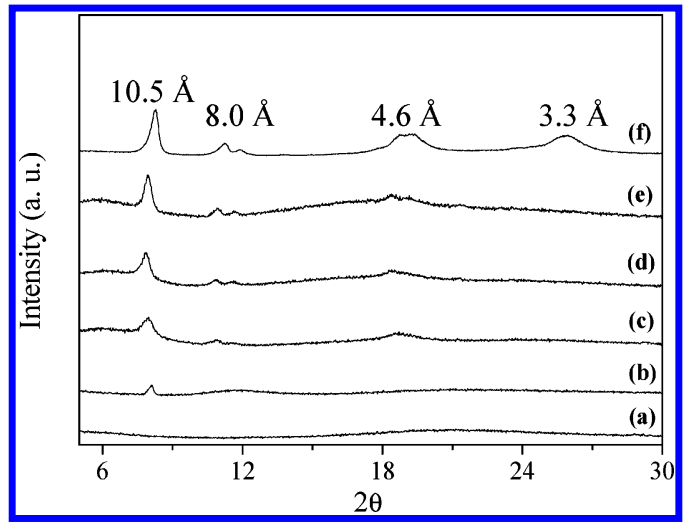

Figure 3. X-ray diffraction curves of PFO-POSS nanocomposite films: (a) PFO, (b) PFO-POSS-1\%, (c) PFO-POSS3\%, (d) PFO-POSS-5\%, (e) PFO-POSS-10\%, and (f) pure Cl-POSS

Table 2. Optical Properties of the PFO-POSS Nanocomposites

\begin{tabular}{|c|c|c|c|c|c|}
\hline & \multicolumn{2}{|c|}{$\lambda_{\max }(\mathrm{UV}, \mathrm{nm})$} & \multicolumn{2}{|c|}{$\lambda_{\max }(\mathrm{PL}, \mathrm{nm})^{a}$} & \multirow{2}{*}{$\begin{array}{l}\text { quantum } \\
\text { yield } \\
\text { film }^{c}\end{array}$} \\
\hline & solution $^{b}$ & film & solution $^{b}$ & film & \\
\hline FO & 38 & 390 & 418 & 425 & 0.55 \\
\hline PFO- & 37 & 383 & 417 & 423 & 0.57 \\
\hline PFO-POS & 374 & 381 & 417 (437) & $423(447)$ & 0.64 \\
\hline PFO-POSS-5\% & 372 & 381 & 417 (437) & $423(447)$ & 0.67 \\
\hline PFO-POSS-10\% & 362 & 380 & $416(436)$ & $422(446)$ & 0.86 \\
\hline
\end{tabular}

${ }^{a}$ The data in parentheses are the wavelengths of shoulders and subpeaks. ${ }^{b}$ The absorption and emission measured in THF. ${ }^{c}$ PL quantum yield estimated relative to a sample of poly $\left(2,7-\left(9,9^{\prime}\right.\right.$ dioctylfluorene) $\left(\Phi_{\mathrm{FL}}=0.55\right)$.

X-ray diffraction peaks for pure PFO. There are three distinct diffraction peaks at $2 \theta=8.3,19.1$, and $26.1^{\circ}$ observed for Cl-POSS (Figure 3f), which correspond to $d$-spacings of $10.5,4.6$, and $3.3 \AA$, respectively. The $d$-spacing of $10.5 \AA$ reflects the size of the Cl-POSS molecules; the other two spacings reflect the rhombohedral crystal structure of POSS molecules. ${ }^{28}$ The presence of a small crystal peak of POSS in PFO indicates a mild degree of aggregation of POSS molecules. The DSC curves of pure $\mathbf{P F O}{ }^{24}$ indicate a value of $T_{\mathrm{g}}$ of $62{ }^{\circ} \mathrm{C}$, a crystallization exothermal peak $\left(T_{\mathrm{c}}\right)$ at $98{ }^{\circ} \mathrm{C}$, and a melting endothermal peak $\left(T_{\mathrm{m}}\right)$ at $155^{\circ} \mathrm{C}$ (see the Supporting Information). Only a small $T_{\mathrm{m}}$ at $166{ }^{\circ} \mathrm{C}$ appears in the curve for PFO-POSS-10\%; the disappearance of the $T_{\mathrm{g}}$ and $T_{\mathrm{c}}$ peaks in this case (PFO containing $10 \%$ POSS ) can be explained by the fact that the mobility of the PFO main chains, including chain folding, is severely retarded by the steric hindrance imposed by the bulky side-chain-tethered POSS units, as has been reported in the literature. ${ }^{25}$ The UV-vis and photoluminescence spectra of PFO and PFO-POSS recorded in THF are presented in the Supporting Information. Table 2 lists the wavelengths of the absorption and PL maxima and the quantum yields of PFO-POSS. The absorption and emission peak maxima of PFO occur at 384 and $418 \mathrm{~nm}$, respectively; these values are close to those reported in the literature. ${ }^{26} \mathrm{We}$ observed no aggregation band in these spectra because THF is a good solvent for PFO. The absortion and emission peaks for PFO and PFO-POSS are almost identical. For each polymer, the absorption peak maximum in solution (THF) is located between 384 and $362 \mathrm{~nm}$, with a slight blue shift caused by the presence of POSS; the PL maxima occur at similar wavelengths for all of the polymers. The quantum yields of the

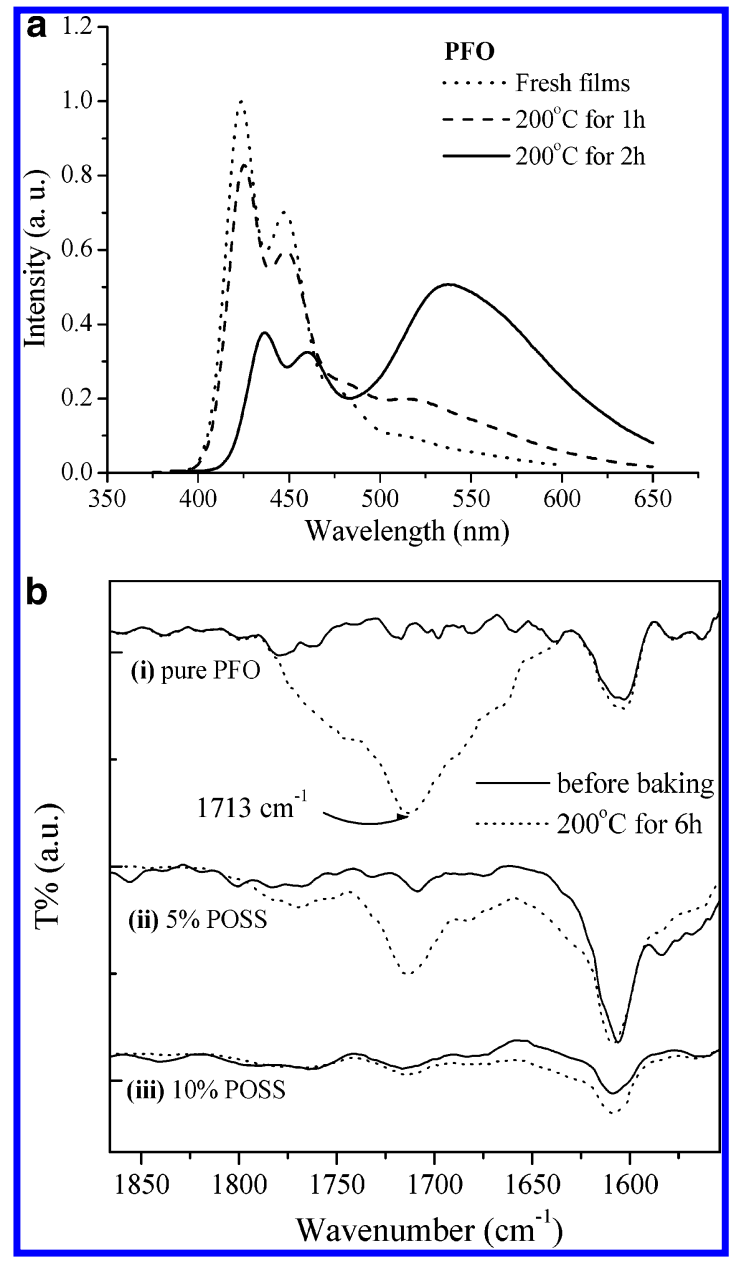

Figure 4. (a) PL spectra of PFO films before annealing (dotted line) and after annealing at $200{ }^{\circ} \mathrm{C}$ for $1 \mathrm{~h}$ (dashed line) and $2 \mathrm{~h}$ (solid line) under a nitrogen atmosphere. (b) FTIR spectra of (i) PFO, (ii) PFO-POSS-5\%, and (iii) PFO-POSS$\mathbf{1 0 \%}$ before (solid line) and after (dashed line) baking at 200 ${ }^{\circ} \mathrm{C}$ for $6 \mathrm{~h}$.

PFO-POSS copolymers increased substantially as the amount of tethered POSS increased. In particular, the quantum yield of PFO containing 10\% POSS was 54\% higher than that of pure PFO (0.86 vs 0.55$)$. This finding can be attributed to the steric hindrance caused by the POSS units preventing aggregation of the PFO main chains, which, in turn, reduces the degree of dimer formation after excitation. This phenomenon is a result of the particular side-chain-tethered POSS architecture that we have employed: it has not been reported in previous studies of POSS/PFO copolymers. The other significant effect that the incorporation of the silsesquioxane into PFO side chain causes is the persistence of luminescence behavior after thermal treatment of PFO-POSS. The stability of both color and luminescence at elevated temperatures are critical for polymer LEDs because sometimes the operating temperature of these devices exceeds $86{ }^{\circ} \mathrm{C}$. Next, we investigated in detail the effect that thermal treatment has on POSSincorporated PFO. Figure 4a displays the photoluminescence spectra of PFO as a solid film after heat treatment at $200{ }^{\circ} \mathrm{C}$; in all cases, the absorption peaks at $386 \mathrm{~nm}$ remain. Other than the main peak at 425 $\mathrm{nm}$, we observe an additional small peak, at ca. $530 \mathrm{~nm}$, in the spectrum of PFO film exposed at $200{ }^{\circ} \mathrm{C}$ for $1 \mathrm{~h}$. The green emission peak at $530 \mathrm{~nm}$ increased in intensity, while the intensity of the main $425 \mathrm{~nm}$ peak 

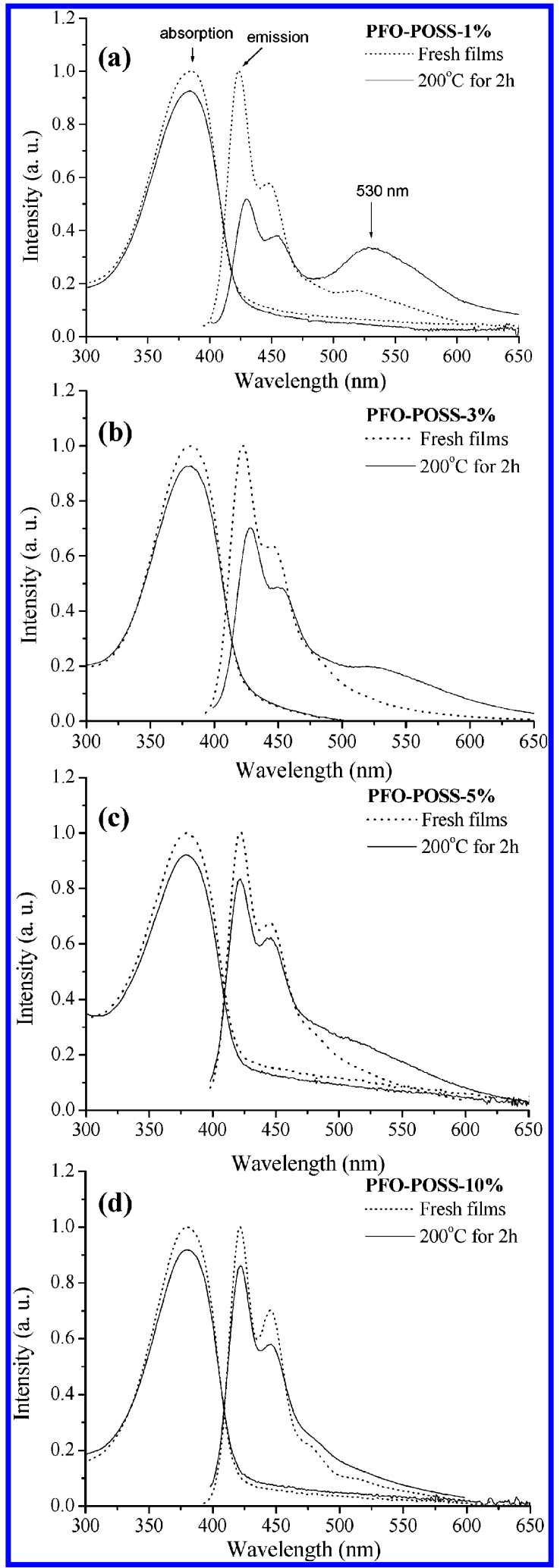

Figure 5. UV-vis absorption and PL spectra of (a) PFO-POSS-1\%, (b) PFO-POSS-3\%, (c) PFO-POSS-5\%, and (d) PFO-POSS-10\% films before (dotted line) and after (solid line) annealing at $200{ }^{\circ} \mathrm{C}$ for $2 \mathrm{~h}$ under a nitrogen atmosphere.

reduced quite dramatically, after annealing the PFO film for $2 \mathrm{~h}$. Figure $4 \mathrm{~b}$ indicates that, after thermal treatment, the intensity of the keto peak $(\mathrm{C}=\mathrm{O}, 1713$ $\mathrm{cm}^{-1}$ ) in the FTIR spectra decreases as the amount of POSS in PFO increases: almost no keto peak appears

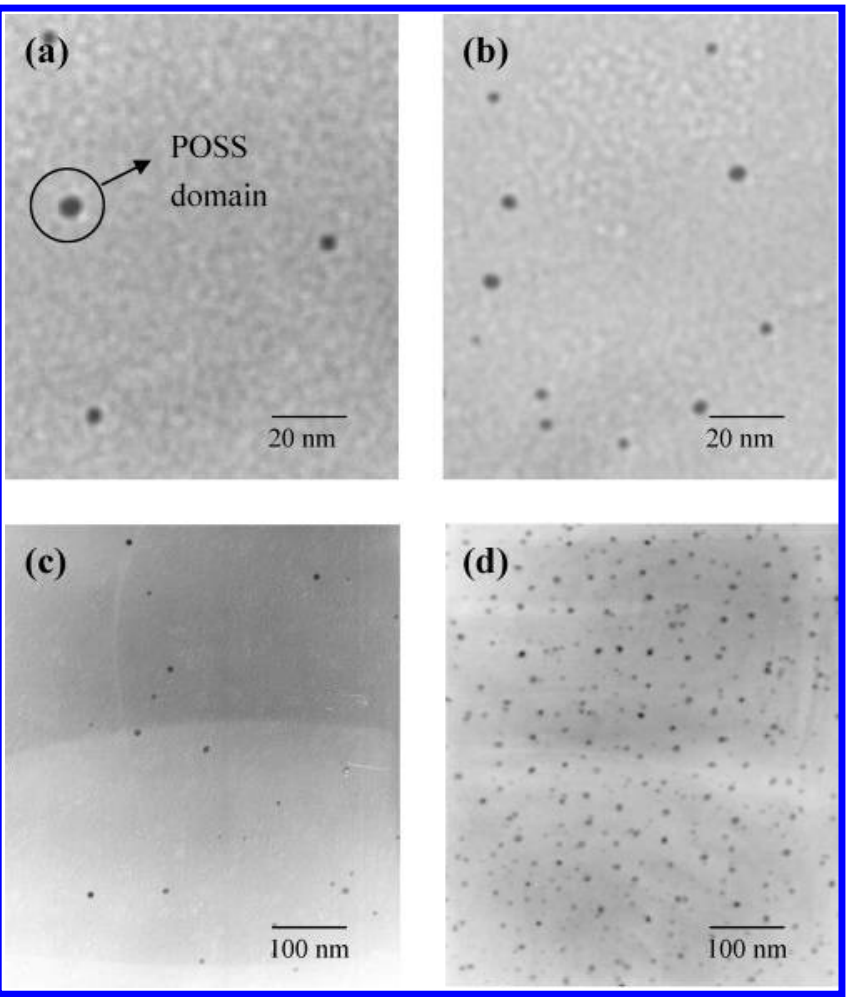

Figure 6. Transmission electron micrographs of (a) PFO-POSS-1\%, (b) PFO-POSS-3\%, (c) PFO-POSS-5\%, and (d) PFO-POSS-10\%.

in the spectrum of the sample containing 10\% POSS. Undesired fluorescence emission resulting from the oxidation defects in polymer chains can be reduced by improving the thermal-oxidation properties of the polymers. ${ }^{18 b, 28}$ In this case, the bulky siloxane units attached to 4-aminophenyl spacer appear to provide a good shield for the neighboring dialkyl groups from thermal oxidation because the thermal degradation temperature of siloxane units is higher than that of dialkyl chains, as evidenced in the TGA results of POSS/PFO copolymer in Table 1, where the thermal degradation temperature of the copolymer increases with the amount of POSS.

Figure 5 presents the normalized absorption and PL emission spectra of the annealed PFO-POSS films with respect to that of their fresh films. The fact that the absorption $\lambda_{\max }$ of PFO-POSS in Figure 5, parts $c$ and $d$ appears at the same wavelength before and after heating suggests that the thermal treatment did not disrupt the conjugation in these PFO-POSS samples. The PL spectrum of PFO containing 1\% POSS exhibits a much smaller shoulder at $530 \mathrm{~nm}$ than that for the pure PFO after the same thermal treatment. This peak was reduced further when 3\% POSS (Figure 5b) was present and became an insignificant shoulder at ca. 500 $\mathrm{nm}$ when $\geq 5 \%$ POSS was incorporated into PFO (Figure 5c,d). These results suggest that the tethered POSS units not only reduced the aggregation of PFO molecular chains but also prevented keto defects from forming upon thermal treatment. Figure 6 presents transmission electron microscopy (TEM) images of different PFO-POSS samples; these images clearly reveal that no large aggregates have formed, but small domains of POSS are present, and the POSS domains are dispersed well in the polymer matrix.

Electroluminescence (EL) Characteristics. Figure 7 displays the electroluminescence (EL) spectra of 


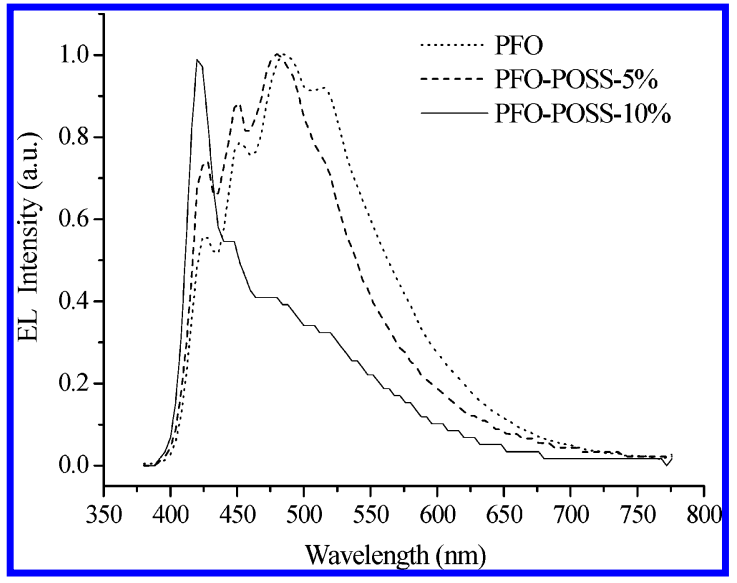

Figure 7. Electroluminescence spectra of the devices prepared from PFO-POSS and PFO in the configuration ITO/ $\mathrm{PEDOT} /$ polymer/Ca/Al.

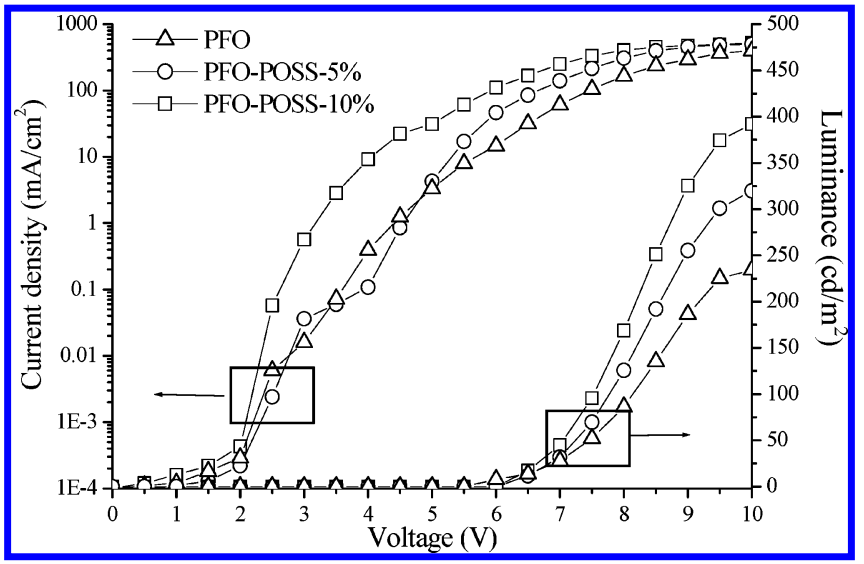

Figure 8. $I-V$ curves of the devices prepared from PFOPOSS and PFO in the configuration ITO/PEDOT/polymer/ $\mathrm{Ca} / \mathrm{Al}$.

PFO-POSS devices. The EL device prepared from PFO emits a weak blue signal at $425 \mathrm{~nm}$ and a more intense green signal in the range $470-600 \mathrm{~nm}$, which is presumably due to the aggregation and keto defects discussed previously. When 5\% POSS was included in PFO, the intensity of the peak at $425 \mathrm{~nm}$ increased, but the intensity of the green emission remained approximately the same. In the case where 10\% POSS was incorporated in PFO, the green emission in the range 470-600 $\mathrm{nm}$ was reduced sharply, while the peak at $425 \mathrm{~nm}$ became the major emission peak and had an intensity much larger than that exhibited by the device prepared from pure PFO. The reduction in the green emission is apparently due to the presence of the siloxane units. The introduction of bulky siloxane units into polyfluorene side chain presumably serves a dual function; it not only hinders oxidation of fluorenes but also increases the interchain distance, thereby retarding the interchain interactions and leading to a reduction of excitons migration to defect sites as discussed in a previous study. ${ }^{27}$

Figure 8 displays the variations of the current density and brightness of the EL devices. The turn-on voltage increased to $5.8 \mathrm{~V}$ forPFO containing $10 \%$ POSS from $5.4 \mathrm{~V}$ for the pure-PFO EL device. A significant increase (68\%) in the maximum brightness of the PFO-POSS10\%-based device occurred relative to that of the purePFO EL device (392 vs $234 \mathrm{~cd} / \mathrm{m}^{2}$ ) at a drive voltage of $10 \mathrm{~V}$ and a current density of $576 \mathrm{~mA} / \mathrm{cm}^{2}$. These improvements might be due to a combination of a lesser degree of aggregation and fewer keto defects being formed upon the incorporation of POSS into PFO.

\section{Summary}

We have synthesized a novel polyfluorene side-chaintethered polyhedral silsesquioxane that has a welldefined architecture. This particular molecular architecture of PFO-POSS increases the quantum yield of polyfluorene significantly by reducing the degree of interchain aggregation; it also results in a purer and stronger blue light being emitted from the EL device by preventing the formation of keto defects.

Acknowledgment. The authors thank the National Science Council, Taiwan, and the US Air Force Office of Scientific Research for funding this work through Grants NSC 91-2120-M-009-001 and AOARD-03-4018, respectively. Mr. Yao-Te Chang is also acknowledged for experimental assistance and helpful discussions.

Supporting Information Available: Figures showing normalized UV-vis absorption, photoluminescence spectra, DSC, and TGA of PFO and PFO-POSS. This material is available free of charge via the Internet at http://pubs.acs.org.

\section{References and Notes}

(1) (a) Greenham, N. C.; Moratti, S. C.; Bradley, D. D. C.; Friend, R. H.; Holmes, A. B. Nature 1993, 365, 628. (b) Gustafsson, G.; Cao, Y.; Treacy, G. M.; Klavetter, F.; Colaneri, N.; Heeger, A. J. Nature 1992, 357, 477. (c) Burroughes, J. H.; Bradley, D. D. C.; Brown, A. R.; Marks, R. N.; Mackay, K.; Friend, R. H.; Burns, P. L.; Holmes, A. B. Nature 1990, 347, 539. (d) Jenekhe, S. A.; Osaheni, J. A. Science 1994, 620, 765.

(2) (a) Halls, J. J. M.; Walsh, C. A.; Greenham, N. C.; Marseglia, E. A.; Friend, R. H.; Moratti, S. C.; Holmes, A. B. Nature (London) 1995, 376, 498. (b) Yu, G.; Gao, J.; Hummelen, J. C.; Wudl, F.; Heeger, A. J. Science 1995, 270, 1789. (c) Granström, M.; Petritsch, K.; Arias, A. C.; Lux, A.; Andersson, M. R.; Friend, R. H. Nature 1998, 395, 257.

(3) (a) Yang, Y.; Heeger, A. J. Nature 1994, 372, 344. (b) Brown, A. R.; Pomp, A.; Hart, C. M.; de Leeuw, D. M. Science 1995, 270, 972. (c) Sirringhaus, H.; Tessler, N.; Friend, R. H. Science 1998, 280, 1741. (d) Sirringhaus, H.; Brown, P. J.; Friend, R. H.; Nielsen, M. M.; Bechgaard, K.; Langeveld-Voss, B. M. W.; Spiering, A. J. H.; Janssen, R. A. J.; Meijer, E. W ; Herwig, P.; de Leeuw, D. M. Nature 1999, 401, 685. (e) Babel, A.; Jenekhe, S. A. J. Am. Chem. Soc. 2003, 125, 13656.

(4) Ranger, M.; Rondeau, D.; Leclerc, M. Macromolecules 1997, 30, 7686 .

(5) Yu, W.-L.; Pei, J.; Cao, Y.; Huang, W.; Heeger, A. J. Chem. Commun. 1999, 1837.

(6) $\overline{P e i, ~ J . ; ~ Y u, ~ W .-L . ; ~ H u a n g, ~ W . ; ~ H e e g e r, ~ A . ~ J . ~ C h e m . ~ C o m m u n . ~}$ 2000, 1631.

(7) Ego, C.; Marsitzky, D.; Becker, S.; Zhang, J.; Grimsdale, A. C.; Müllen, K.; MacKenzie, J. D.; Silva, C.; Friend, R. H. J. Am. Chem. Soc. 2003, 125, 437.

(8) (a) Lee, J. I.; Klärner, G.; Miller, R. D. Chem. Mater. 1999 11, 1083. (b) Klärner, G.; Lee, J. I.; Davey, M. H.; Miller, R. D. Adv. Mater. 1999, 11, 115.

(9) (a) Yu, W.-L.; Pei, J.; Huang, W.; Heeger, A. J. Adv. Mater 2000, 12, 828. (b) Zeng, G.; Yu, W.-L.; Chua, S.-J.; Huang, W. Macromolecules 2002, 35, 6907.

(10) Setayesh, S.; Grimsdale, A. C.; Weil, T.; Enkelmann, V.; Müllen, K.; Meghdadi, F.; List, E. J. W.; Leising, G. J. Am. Chem. Soc. 2001, 123, 946.

(11) (a) Kreyenschmidt, M.; Klärner, G.; Fuhrer, T.; Ashenhurst, J.; Karg, S.; Chen, W. D.; Lee, V. Y.; Scott, J. C.; Miller, R. D. Macromolecules 1998, 31, 1099. (b) Klärner, G.; Lee, J. I.; Lee, V. Y.; Chan, E.; Chen, J. P.; Nelson, A.; Markiewicz, D.; Siemens, R.; Scott, J. C.; Miller, R. D. Chem. Mater. 1999, 11, 1800. (c) Lee, J. I.; Klärner, G.; Miller, R. D. Chem. Mater. 1999, 11, 1083 .

(12) (a) Marsitzky, D.; Klapper, M.; Mullen, K. Macromolecules 1999, 32, 8685. (b) Marsitzky, D.; Murray, J.; Scott, J. C.; Carter, K. R. Chem. Mater. 2001, 13, 4285. (c) Marsitzky, D.; Vestberg, R.; Blainey, P.; Tang, B. T.; Hawker, C. J.; 
Carter, K. R. J.Am. Chem. Soc. 2001, 123, 6965. (d) Setayesh, S.; Grimsdale, A. C.; Weil, T.; Enkelmann, V.; Müllen, K.; Meghdadi, F.; List, E. J. W.; Leising, G. J. Am. Chem. Soc. 2001, 123, 946. (e) Ego, C.; Grimsdale, A. C.; Uckert, F.; Yu, G.; Srdanov, G.; Müllen, K. Adv. Mater. 2002, 14, 809. (f) Pogantsch, A.; Wenzl, F. P.; List, E. J. W.; Leising, G.; Grimsdale, A. C.; Müllen, K. Adv. Mater. 2002, 14, 1061. (g) Lupton, J. M.; Schouwink, P.; Keivanidis, P. E.; Grimsdale, A. C.; Müllen, K. Adv. Funct. Mater. 2003, 13, 154.

(13) Shu, C. F.; Dodda, R.; Wu, F. I.; Liu, M. S.; Jen, A. K. Y. Macromolecules 2003, 36, 6698.

(14) Zeng, G.; Yu, W. L.; Chua, S. J.; Huang, W. Macromolecules 2002, 35, 6907.

(15) Xiao, S.; Nguyen, M.; Gong, X.; Cao, Y.; Wu, H.; Moses, D.; Heeger, A. J. Adv. Funct. Mater. 2003, 13, 25

(16) (a) Cho, H. J.; Jung, B. J.; Cho, N. S.; Lee, J.; Shim, H. K. Macromolecules 2003, 36, 6704. (b) Lim, E.; Jung, B. J.; Shim, H. K. Macromolecules 2003, 36, 4288.

(17) Kulkarni, A. P.; Jenekhe, S. A. Macromolecules 2003, 36, 5285.

(18) (a) List, E. J. W.; Guentner, R.; Freitas, P. S.; Scherf, U. Adv. Mater. 2002, 14, 374. (b) Romaner, L.; Pogantsch, A.; Freitas, P. S.; Scherf, U.; Gaal, M.; Zojer, E.; List, J. W. Adv. Funct. Mater. 2003, 13, 597. (c) Kulkarni, A. P.; Kong, X.; Jenekhe, S. A. J. Phys. Chem. B. 2004, 108, 8689. (d) Cho, H. J.; Jung, B. J.; Cho, N. S.; Lee, J.; Shim, H. K. Macromolecules 2003 , 36, 6704. (e) Lin, W. J.; Chen, W. C.; Wu, W. C.; Niu, Y. H.; Jen, A. K. Y. Macromolecules 2004, 37, 2335.

(19) (a) Leu, C. M.; Chang, Y. T.; Wei, K. H. Macromolecules 2003, 36, 9122. (b) Kraft, A.; Grimsdale, A. C.; Holmes, A. B. Angew.
Chem., Int. Ed. Engl. 1998, 37, 402.

(20) Setayesh, S.; Grimsdale, A. C.; Weil, T.; Enkelmann, V.; Müllen, K.; Meghdadi, F.; List, E. J. W.; Leising, G. J. Am. Chem. Soc. 2001, 123, 946.

(21) Miller, T. M.; Neenan, T. X.; Zayas, R.; Bair, H. E. J. Am. Chem. Soc. 1992, 114, 1018.

(22) Ranger, M.; Leclerc, M. Macromolecules 1999, 32, 3306.

(23) Leu, C. M.; Chang, Y. T.; Wei, K. H. Chem. Mater. 2003, 15, 3721.

(24) Grell, M.; Bradley, D. D. C.; Inbasekaran, M.; Woo, E. P. $A d v$. Mater. 1997, 9, 798.

(25) Wu, F. I.; Reddy, D. S.; Shu, C. F.; Liu, M. S.; Jen, A. K. Y. Chem. Mater. 2003, 15, 269.

(26) (a) Grell, M.; Bradley, D. D. C.; Long, X.; Chamberlain, T.; Inbasekaran, M.; Woo, E. P.; Soliman, M. Acta Polvm. 1998, 49, 439. (b) Grice, A. W.; Bradley, D. D. C.; Bernius, M. T.; Inbasekaran, M.; Wu, W. W.; Woo, E. P. Appl. Phvs. Lett. 1998, 73, 629. (c) Friend, R. H.; Gymer, R. W.; Holmes, A. B.; Burroughes, J. H.; Marks, R. N.; Taliani, C.; Bradley, D. D. C.; Dos Santos, D. A.; Bredas, J. L.; Logdlund, M.; Salaneck, W. R. Nature 1999, 397, 121.

(27) Grimsdale, A. C.; Leclère, P.; Lazzaroni, R.; MacKenzie, J. D.; Murphy, C.; Setayesh, S.; Silva, C.; Friend, R. H.; Müllen, K. Adv. Funct. Mater. 2002, 12, 729 .

(28) Zheng, L.; Waddon, A. J.; Farris, R. J.; Coughlin, E. B. Macromolecules 2002, 35, 2375.

MA0479520 